\title{
The Small Round Blue Cell Tumors of the Sinonasal Area: Histological and Immunohistochemical Findings
}

\author{
Mohammad J Ashraf ${ }^{1}$, Leila Beigomi ${ }^{1}$, Negar Azarpira ${ }^{1,}$, , Bita Geramizadeh ${ }^{1}$, Bijan \\ Khademi $^{2}$, Afsoon Hakimzadeh ${ }^{1}$, Elham Abedi ${ }^{1}$ \\ 1 Department of Pathology, Shiraz Medical School, Shiraz University of Medical Sciences, Shiraz, IR Iran \\ 2 Department of Otolaryngology, Shiraz Medical School, Shiraz University of Medical Sciences, Shiraz, IR Iran \\ ${ }^{*}$ Corresponding author: Negar Azarpira, Department of Pathology, Shiraz Medical School, Shiraz University of Medical Sciences, Shiraz, IR Iran. Tel: +98-711647433, Fax:+98-7116474331, \\ E-mail: negarazarpira@ yahoo.com.
}

Received: March 04, 2012; Accepted: April 26, 2013

\begin{abstract}
Background: Primary Small round blue cell tumors (SRBCT) in sinonasal comprise histogenetically diverse entities with overlapping morphologic features. Because of the limited initial biopsy tissue materials, differential diagnostic difficulties may arise, and as they have different management, exact diagnosis and classification are very important.

Objectives: In this study, we analyzed the immunohistochemical expression of a panel of markers in the classification and diagnosis of sinonasal SRBCTs.

Material and Methods: This cross sectional study was performed on 36 paraffin embedded tissue samples. Histologic and immunohistochemical slides from 36 patients with SRBCT were analyzed retrospectively. The patients were admitted in Khalili hospital, Shiraz from 1383 to 1388.

Results: There were 13 women and 23 men with the mean age of $53 \pm 12.1$. There were 9 malignant melanoma, seven poorly differentiated SCC; six lymphoma (DLBL); 4 SCNEC; three SNUC; two ON; two Ewing/PNET; two embryonal rhabdomyosarcoma, and one plasmacytoma. Pan-cytokeratin was strongly expressed poorly differentiated SCC and all cases of SNUC. Coexpression of desmin and nuclear myoD1 was only detected in rhabdomyosarcoma. HMB45 was only expressed in sinonasal melanoma. CD99 expression was identified only in Ewing/ PNET. FLI-1 was detected in 50\% of PNET. P63 was expressed in poorly differentiated SCC (2/7) and SNUC (1/3).

Conclusions: The results of our study indicate that the integration of histopathologic findings with application of limited but highly specific markers led to the separation of carcinomas, lymphoma and melanomas from other small cell tumors. Using a panel of keratin, LCA, desmin, and HMB45 is the most practical and economic approach to accurately classify these tumors.
\end{abstract}

Keywords: Carcinoid Tumor; IHC64; Histological Techniques

\section{Background}

The sinonasal regions are host to a variety of malignant neoplasms. Approximately $0.2-0.8$ percent of all malignant tumors occur in nasal cavity and paranasal sinuses, and because of the close anatomic relation with the orbits and skull base, disease extension into these structures usually occurs $(1,2)$. The most common locations are maxillary sinus, followed by the nasal cavity, the ethmoid, sphenoid, and frontal sinuses $(1,2)$. The "small round blue cell tumors" (SRBCTs) constitute a heterogeneous group of malignant neoplasms characterized by a monomorphic population of undifferentiated cells with small-sized nuclei and scant cytoplasm (1). An early and accurate diagnosis is imperative for appropriate treatment. However, definitive diagnosis of SRBCT based solely on the $\mathrm{H} \& \mathrm{E}$ light microscopic findings may be difficult. Furthermore, pathologist usually receives a small or limited size biopsy, which complicates the diagnosis. Ancillary studies such as immunohistochemistry (IHC), cytogenetic and molecular techniques may be used to roll out differential diagnoses of SRBCTs. The SRBCTs of sinonasal area are categorized as:

1. Epithelial SRBCTs including Poorly Differentiated, Nonkeratinizing Squamous Cell Carcinoma, Sinonasal Undifferentiated Carcinoma (SNUC), Small Cell Carcinoma, and Neuroendocrine Type (SCCNET) $(1,3,4)$. Squamous cell carcinoma (SCC) is the most common malignancy of the sinonasal tract. Well-differentiated and/or keratinizing form of SCC is easily recognizable but the poorly differentiated, non-keratinizing variant may exhibit histopathologic features that overlap with other SRBCTs. Finding of an in situ carcinoma and/or direct

Implication for health policy/practice/research/medical education:

In this study, we analyzed the immunohistochemical expression of a panel of markers in the classification and diagnosis of sinonasal SRBCTs.

Copyright (c) 2013, Iranian Red Crescent Medical Journal; Licensee Kowsar Ltd. This is an Open Access article distributed under the terms of the Creative Commons Attribution License (http://creativecommons.org/licenses/by/3.0), which permits unrestricted use, distribution, and reproduction in any medium, provided the original work is properly cited. 
continuity of neoplastic cells to the overlying surface epithelium are useful histological finding in favor of epithelial origin. Immunohistochemically, pan cytokeratin, $\mathrm{CK} 7, \mathrm{CK} 8$, and EMA immunoreactivity are useful for distinguishing this neoplasm from other small cell tumors $(1,3,4)$. SNUC is a rare, highly aggressive carcinoma which typically presents with locally extensive disease $(1,2)$. Histopathologically, the tumor cells grow along the mucosal surface epithelium with extension into superficial mucosal glands $(1,2)$. Individual malignant cells exhibit hyperchromatic to vesicular nuclei with high nuclearto-cytoplasmic ratio and prominent nucleoli. Immunohistochemically, the tumor cells are immunoreactive for pan-cytokeratins and simple keratins with no amplification of Epstein-Barr virus (EBV) RNA by in situ hybridization $(1,3,4)$. SCCNETs are composed of small sized cells with oval or round hyperchromatic nuclei and absent or inconspicuous nucleoli, arranged in sheets, nests, and/ or trabeculae. Crush artifact with a high mitotic rate is a common finding $(1,2,5)$. Punctuate perinuclear cytokeratin staining is an important finding. CD56 staining is also common $(1,5)$.

2. Neuroectodermal SRBCTs including Olfactory Neuroblastoma (ON), Sinonasal Mucosal Malignant Melanoma, and Extraskeletal Ewing's sarcoma/Primitive Neuroectodermal Tumor (ES/PNET). Olfactory neuroblastoma (ON) is uncommon, accounting for only $1 \%$ to $5 \%$ of malignant nasal cavity neoplasms (6-8). ON is thought to originate from the olfactory portion of the mucous membrane lining the nasal fossa and is virtually confined to the upper nasal cavity in the region of the cribriform plate. The presence of fibrillary cell processes, Homer Wright rosettes, and S100-positive sustentacular cells are histological findings in favor of ON. In contrast to PNET, ON is not immunoreactive for $\operatorname{CD} 99(6,8)$. Amelanotic variant with small cell morphology of malignant melanomas is prone to misclassification with other SRBCTs of this area. Melanin pigment is observed in approximately $2 / 3$ of cases ( 7 , 9). Diffuse staining for S-100 and HMB45 are useful for final diagnosis $(1,7,10)$. CD99 immunoreactivity is useful in distinguishing ES/PNET from most other SRBCTs. Identification of the characteristic $t(11 ; 22)(q 24 ; q 12)$ or EWSR1-FL1 fusion transcript or defined variant translocation can be invaluable in confirming this diagnostic entity (11).

3. Mesenchymal SRBCTs including Rhabdomyosarcoma, Poorly Differentiated Synovial Sarcoma. Both embryonal and alveolar rhabdomyosarcoma frequently occur in the sinonasal area $(1,2)$. Rhabdomyosarcoma usually express myogenic markers such as MyoD1 and desmin. However aberrant staining for CK and synaptophysin is reported. Distinguishing from SCCNET or SNUC is possible with identification of the 2;13 and 1;13 translocations or respective PAX3-FOXO1 and PAX7-FOXO1 fusion transcripts $(1,2)$. Poorly Differentiated Synovial Sarcoma rarely occurs in sinonasal tract. Immunoreactivity for cytokeratin, EMA, BCL2, coupled with cytogenetic or FISH confirmation of the $t(X ; 18)$ (p11.2;q11.2) or derived SYT-SSX chimeric transcripts facilitates the diagnosis $(1,2,12)$.

4. Hematolymphoid SRBCTs including Extramedullary Plasmacytoma, Extranodal NK/T Cell Lymphoma (NasalType) and Diffuse large B cell lymphoma (DLBL) $(1,13)$. In respect of extramedullary plasmacytoma, when tumor is mainly composed of mature plasma cell the diagnosis is not challenging. But in poorly differentiated tumors (immature plasma cells) with occasional EMA or cytokeratin positivity, misdiagnosed as a carcinoma may occur. The second most common malignancy of the sinonasal tract following SCC is malignant lymphoma $(13,14)$. Extranodal NK/T Cell Lymphoma (Nasal-Type) frequently affects Asian and Latin American populations (13). Demonstration of the EBV virus (EBV-encoded early RNAs) by in situ hybridization in addition to a NK-cell immunophenotype (CD3-, CD56+, perforin and granzyme B+) confirmed the diagnosis (13-15). DLBL is typically more common in Western populations. Immunophenotypically, DLBL has positive findings for B-lineage markers (CD20+, CD79+, CD3-, CD56) $(1,12)$.

\section{Objectives}

In this study, we analyzed the immunohistochemical expression of a panel of markers in the classification and diagnosis of sinonasal SRBCTs.

\section{Patients and Methods}

We retrieved 36 cases of SRBCT from division of surgical pathology archives at the Shiraz University of Medical Sciences, between 2006 and 2011. All hematoxylin and eosin-stained and immunohistochemical slides of these tumors were available and reevaluated independently by two head and neck pathologists. The final diagnoses were rhabdomyosarcoma, olfactory neuroblastoma, Ewing sarcoma, sinonasal undifferentiated carcinomas (SNUCs), neuroendocrine carcinomas, and sinonasal melanomas. No original diagnoses were changed on rereview. Table 1 presents the markers used and the conditions for the IHC staining procedure. Immunohistochemical markers included known lineage-specific markers (pan cytokeratin AE1/AE3, S100, HMB45, desmin, and myoD1), basal cell marker P63, markers of neuroendocrine differentiation (chromogranin, synaptophysin, and NSE), markers for lymphoma (leukocyte common antigen (LCA), CD20, CD3). The FLI-1, product of chromosomal translocation between the FL1 gene and EWS gene, are considered for ancillary marker of Ewing/PNET Markers were evaluated for cytoplasmic staining except for P63, S100, LIF-1 and myoD1, which had positive results for nuclear expression, and CD99, for which membranous staining was considered. 
JAshrafM et al.

\begin{tabular}{|c|c|c|c|c|c|}
\hline Manufacture & Chromogene & Retrieval & Dilution & Clone & Biomarker \\
\hline Dako, Denmark & DAB, Envision & Citrate & Ready to use & AE1/AE3 & $C K$ \\
\hline Dako, Denmark & DAB, Envision & Citrate & Ready to use & - & $\mathrm{S} 100$ \\
\hline Novacastra & DAB, Envision & Citrate & $1 / 50$ & - & HMB45 \\
\hline Dako, Denmark & DAB, Envision & Citrate & Ready to use & V9 & Vimentin \\
\hline Dako, Denmark & DAB, Envision & Citrate & $1 / 100$ & 2B11 & $L C A$ \\
\hline Dako, Denmark & DAB, Envision & Citrate & $1 / 75$ & D33 & Desmin \\
\hline Dako, Denmark & DAB, Envision & Citrate & $1 / 200$ & $5.8 \mathrm{~A}$ & MyoD1 \\
\hline Dako, Denmark & DAB, Envision & Citrate & Ready to use & $\mathrm{BBs} / \mathrm{NC}$ & NSE \\
\hline Dako, Denmark & DAB, Envision & $\mathrm{TE}$ & Ready to use & - & Chromogranin \\
\hline Dako, Denmark & DAB, Envision & Citrate & Ready to use & SY38 & Synaptophysin \\
\hline Dako, Denmark & DAB, Envision & Citrate & $1 / 200$ & $12 \mathrm{E} 7$ & CD99 \\
\hline Novacastra & DAB, Envision & Citrate & $1 / 100$ & - & P63 \\
\hline Santa Cruz, Santa Cruz, CA, USA & DAB, Envision & Citrate & $1 / 400$ & sc-356 & LFI-1(C-19) \\
\hline
\end{tabular}

\section{Results}

\subsection{Age and Sex Distribution}

There were 13 women and 23 men, age range from 2 to 84 years, with a median age of 53 years. The peak incidence was during the sixth and seventh decades of life in both sexes. Men represented a higher proportion of tumors than women, with male/female ratio of 2/1.

\subsection{Histologic Distribution}

There were nine malignant melanoma, seven poorly differentiated SCC, six lymphoma (DLBL), four SCNEC, three SNUC, two ON, two Ewing/PNET, two embryonal rhabdomyosarcoma, and one plasmacytoma (Table 1) (Figure 1A-E).

Figure1 Histological Comparison of Small Round Blue Cell Tumors in Sinonasal Area
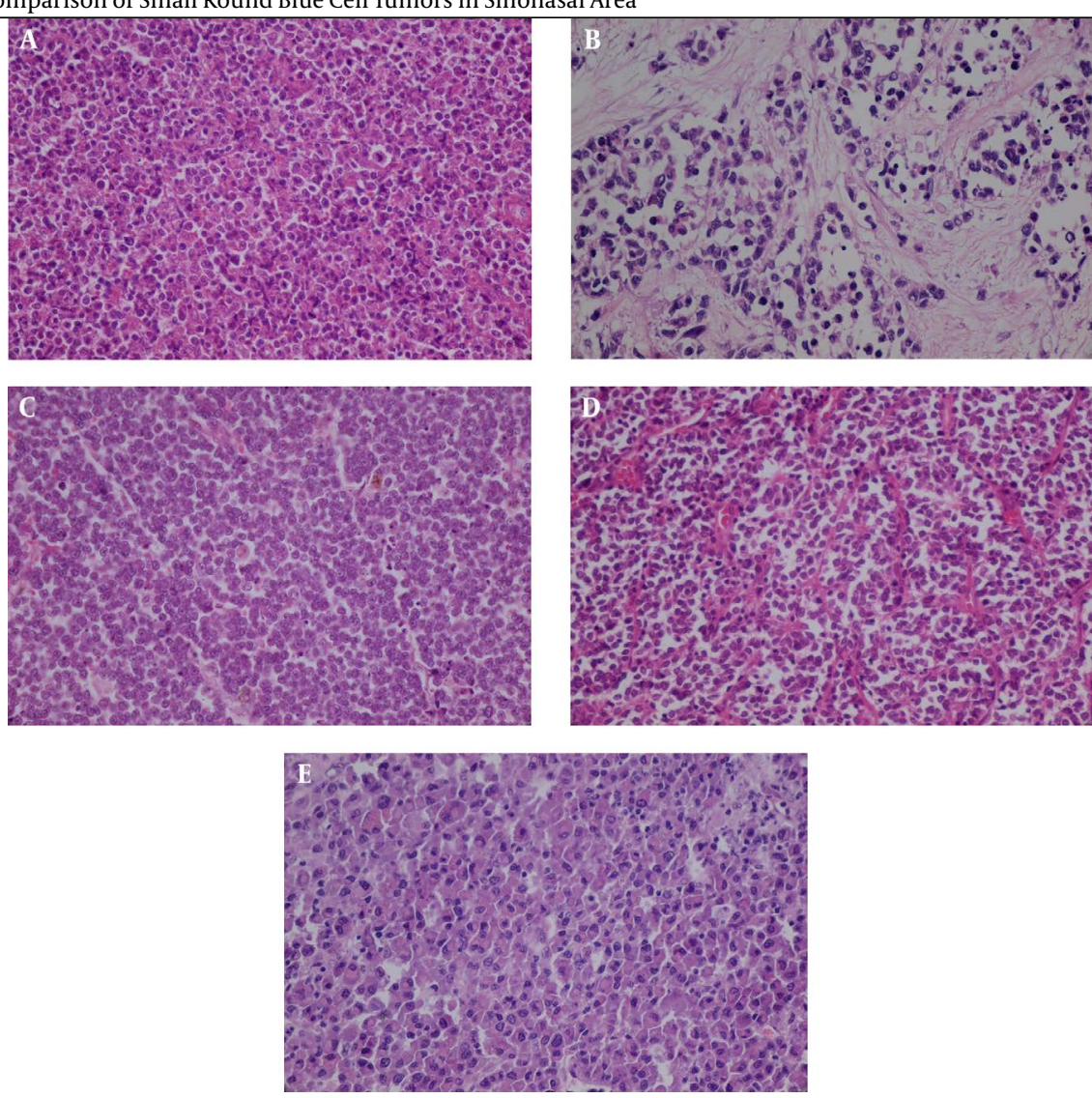

A: Lymphoma, B: Rhabdomyosarcoma, C: Melanoma, D: PNET, E: Plasmacytoma 


\subsection{Immunohistochemical Analysis}

The IHC staining results for sinonasal tumors are presented in Table 2 and patterns of expression were shown in Figure $2 \mathrm{~A}-\mathrm{C}$
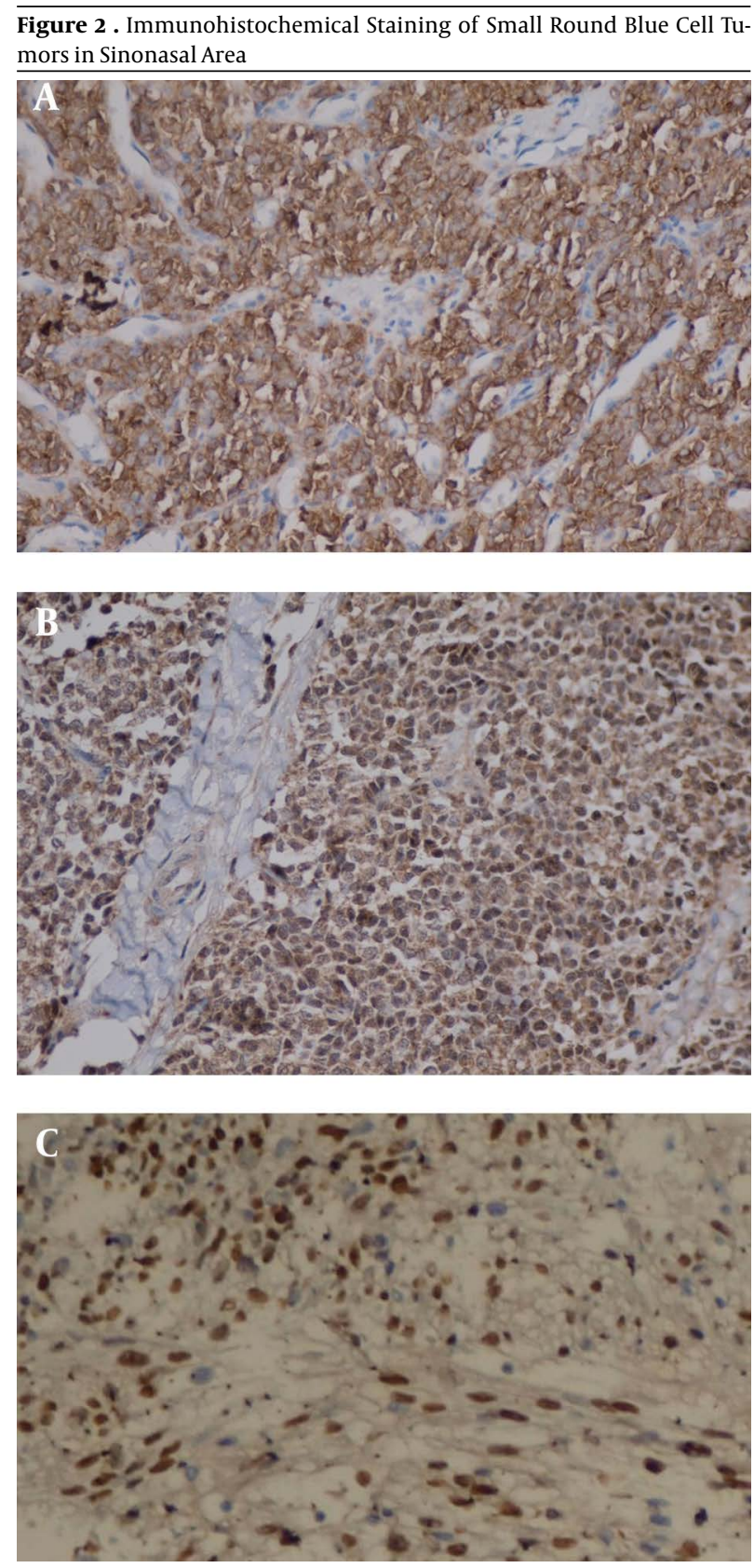

A: Membranous Staining of CD99 in PNET, B: Nuclear FLi-1 Staining in PNET, C: Desmin Expression in Rhabdomyosarcoma

\subsection{A: Epithelial Markers}

Pan-cytokeratin was strongly expressed in 12 (33\%) of the 36 tumors; included in all cases poorly differentiated SCC (7/7), all SNUCs (3/3), and 2 of 4 SCNEC.

\subsection{B: Neuroendocrine Markers}

Neuroendocrine markers (chromogranin, synaptophysin and NSE) were collectively expressed in 12 (33.3\%) of the 36 tumors. Synaptophysin and chromogranin had positive findings in all 2 SCNEC and 1 of 2 ON. NSE expression was noted in 2 of 2 ON, and 3 of 4 SCNEC. Coexpression of two or three of these markers was found in most neuroblastomas and SCNEC.

\subsection{C: Skeletal Muscle Markers}

Of the 36 tumors, 2 (5.5\%) had positive results for desmin and coexpressed nuclear myoD1, confirmed the diagnosis of rhabdomyosarcoma.

\subsection{D: Melanocytic Markers}

S100 was expressed in 10 (28\%) of 36 tumors. Expression was identified in 8 of 9 sinonasal melanomas, and showed focally dispersed positive cells in PNET (1/2). HMB45 was only expressed in sinonasal melanomas (9/9).

\subsection{E: Lymphoma Markers}

LCA and CD20 were expressed in 6 (16.7\%) of 36 tumors. All of them were diagnosed as non-Hodgkin lymphoma, BLBL with further IHC markers.

\subsection{F: Miscellaneous New Markers}

CD99 is a sialomucin glycoprotein adhesion molecule, and is used as a marker in the diagnosis of Ewing/PNET tumors. CD99 expression was identified only in Ewing/ PNET tumors (2/2). FLI-1, a marker for Ewing sarcoma, was present in 2 (5.5\%) of 36 tumors. It was expressed in Ewing/PNET (1/2), and 1 of 6 lymphoma. The P63, a marker for primitive basal cells, was present in 3 (8.3\%) of 36 tumors. It was expressed in poorly differentiated SCC (2/7) and 1 of 3 SNUC.

\section{Discussion}

SRBCTs of the sinonasal tract are rare tumors with a wide spectrum of biological activity and diverse clinical behavior. In the present study, these neoplasms usually occurred in the sixth and seventh decades of life, and rarely found in young age group. This finding was consistent with the results of the literature review in which the average median age of patients in the reported series was 57 years (Table 2$)(12,15,16))$. In our series, the male/ female ratio was $2 / 1$. In the literature review, it was mentioned that men represented a higher proportion of malignant neoplasms than women, with male/female ratio of 1.8 (range from 1.2 to 5.3) (17-20). The combined light microscopic rereview immunophenotyping and molecular findings led to the confirmation of the diagnosis in all 
JAshraf M et al.

\begin{tabular}{|c|c|c|c|c|c|c|c|c|c|c|c|c|c|c|}
\hline FLI-1 & P63 & CD99 & Synapto $^{a}$ & Chromo $^{\mathrm{a}}$ & NSE & MyoD1 & Desmin & LCA & Vimentin & $\mathbf{C K}^{\mathrm{a}}$ & HMB45 & S100 & Number & Tumor type \\
\hline $0 / 9$ & $0 / 9$ & $0 / 9$ & $0 / 9$ & $0 / 9$ & $0 / 9$ & $0 / 9$ & $0 / 9$ & $0 / 9$ & $0 / 9$ & $0 / 9$ & $9 / 9$ & $8 / 9$ & $9(27)$ & Sinonasal melanoma \\
\hline $\mathbf{0} / 7$ & $2 / 7$ & $0 / 7$ & $0 / 7$ & $0 / 7$ & $0 / 7$ & $0 / 7$ & $0 / 7$ & $0 / 7$ & $0 / 7$ & $7 / 7$ & $0 / 7$ & $0 / 7$ & $7(19.4)$ & $\begin{array}{l}\text { Poorly differentiated } \\
\text { SCC }\end{array}$ \\
\hline $1 / 6$ & $0 / 6$ & $0 / 6$ & $0 / 6$ & $0 / 6$ & $0 / 6$ & $0 / 6$ & $0 / 6$ & $6 / 6$ & $0 / 6$ & $0 / 6$ & $0 / 6$ & $0 / 6$ & $6(16.7)$ & Lymphoma \\
\hline $0 / 4$ & $0 / 4$ & $0 / 4$ & $4 / 4$ & $4 / 4$ & $3 / 4$ & $0 / 4$ & $0 / 4$ & $0 / 4$ & $2 / 3$ & $2 / 4$ & $0 / 4$ & $0 / 4$ & $4(11.1)$ & SCNEC $^{\mathrm{a}}$ \\
\hline $\mathbf{0} / 3$ & $1 / 3$ & $0 / 3$ & $0 / 3$ & $0 / 3$ & $0 / 3$ & $0 / 3$ & $0 / 3$ & $0 / 3$ & $0 / 3$ & $3 / 3$ & $0 / 3$ & $0 / 3$ & $3(8.3)$ & SNUC $^{\mathrm{a}}$ \\
\hline $\mathbf{0} / 2$ & $0 / 2$ & $0 / 2$ & $1 / 2$ & $1 / 2$ & $2 / 2$ & $0 / 2$ & $0 / 2$ & $0 / 2$ & $0 / 2$ & $0 / 2$ & $0 / 2$ & $0 / 2$ & $2(5.6)$ & $\mathrm{ON}^{\mathrm{a}}$ \\
\hline $1 / 2$ & $0 / 2$ & $2 / 2$ & $0 / 2$ & $0 / 2$ & $0 / 2$ & $0 / 2$ & $0 / 2$ & $0 / 2$ & $2 / 2$ & $0 / 2$ & $0 / 2$ & $1 / 2$ & $2(5.6)$ & Ewing/PNET \\
\hline $\mathbf{0} / 2$ & $0 / 2$ & $0 / 2$ & $0 / 2$ & $0 / 2$ & $0 / 2$ & $2 / 2$ & $2 / 2$ & & $2 / 2$ & $0 / 2$ & $0 / 2$ & $0 / 2$ & $2(5.6)$ & Embryonal rhabdo \\
\hline $\mathbf{0} / \mathbf{1}$ & $0 / 1$ & $0 / 1$ & $0 / 1$ & $0 / 1$ & $0 / 1$ & $0 / 1$ & $0 / 1$ & $1 / 1$ & $0 / 1$ & $0 / 1$ & $0 / 1$ & $0 / 1$ & $1(2.8)$ & Plasmacytoma \\
\hline
\end{tabular}

a abbreviations: Chromo, chromogranin; CK, cytokeratin; Synapto, synaptophysin; SNUC, sinonasal undifferentiated carcinoma. SCNEC, Small cell neuroendocrine carcinoma; ON, Olfactory neuroblastoma Final diagnoses based on immunohistochemical and morphologic assessment.

of the 36 tumors. Aberrant expression of certain histogenetic marker was not detected in this study. Epithelial tumors (carcinoma) constitute most of sinonasal neoplasms. In the literature, carcinomas composed of differentiated SCC, nonkeratinizing poorly differentiated SCC, SNUC and SCCNET $(1,2,16,21)$. However, in our study, carcinomas constituted $39 \%$ of all sinonasal SRBCTs and differentiated SCC was not considered at all. Overexpression of P63 was considered as a marker for squamous differentiation (21). However, better differentiated areas and even well-differentiated tumors may be negative (10, 21,22 ). In our study, P63 was founded in two of seven cases of poorly differentiated SCC. In the study performed on sinonasal SRBCTs by Wooff et al. (23), 63 had positive results in all cases of nonkeratinizing, poorly differentiated SCC ( 2 of 2 ), and in single cases of mantle cell lymphoma (1 of 1) and poorly differentiated neuroendocrine carcinoma (1 of 1); however, it inconsistently stained diffuse large B-cell lymphoma (4 of 5), extranodal NK/T-cell lymphoma, nasal type (1 of 4), sinonasal undifferentiated carcinoma (1 of 6), and Ewing sarcoma/primitive neuroectodermal tumor ( 2 of 6 ). These differences may be explained by variability at two phases of the procedure: (1) the antibody may not detect all P63 isoforms (2), the difference in interpretation. The first possibility is less likely. Because most of the IHC studies use antibodies that detect all P63 isoforms (TAp63 $\alpha$, TAp63 $\beta$, TAp63 $\gamma, \Delta$ Np63 $\alpha$, $\Delta \mathrm{Np} 63 \beta, \Delta \mathrm{Np} 63 \gamma)(10,21,23,24)$. In agreement with other authors, we considered a positive result when equal or more than $50 \%$ of tumor cells was intensely stained with antibody (21). This discrepancy could be attributed to the use of a small core of tissue and the variable processing differences of the assembled blocks. In the Ewing case, the initial diagnosis was most likely based on the detection of CD99 in tumor cells (25). But, nonspecific diagnostic nature of CD99 was reported in several tumor studies (25 - 27). In our study, no aberrant CD99 expression was found and diffuse strong membranous staining was detected only in Ewing/PNET. Combination of FLI-1 poly- clonal (FLI-1p) and CD99 was considered as a valuable immunohistochemical approach for the diagnosis of EWS / PNET. Mhawech-Fauceglia et al., believed that the best antibody combination for the diagnosis of EWS / PNET was CD99 and FLI-1p (C-19, polyclonal, Santa Cruz, USA), with a very high sensitivity $(26,27)$. However, these two markers can be expressed in other neoplasms, including vascular tumors and Merkel cell carcinoma of the skin (28). We had only two cases of Ewing/PNET, both of them had positive results for CD99, but the LFI-1 was stained in one case. Due to low case number, the correlation between CD99 and FLI-1p was not possible. The application of molecular analysis of the EWS-FLI1 fusion transcript was suggestive of Ewing sarcomas and PNET confirmation. Melanoma was the major malignancy in our case, more than reported frequency by Cordes et al. (16). Like previous studies, our data showed a high incidence of S100 expression in sinonasal melanomas (29). The S100 was not expressed in one case; therefore, we advocate concurrent use of other melanoma markers (HMB-45, Melan- A). Similarly, S100 may also play a role in the diagnosis of ON, but no expression was found in our two cases. Therefore it must be used in conjunction with other complementary markers (30). Lymphomas, manifested as infiltrative sheet-like growth of CD45 positive tumor cells and easily separated for other sinonasal small cell tumors $(2,14)$. The results of our study indicate that the integration of histopathologic findings with a panel of selected lineage specific markers is necessary for early diagnosis and classification of SRBCTs of sinonasal area. The initial application of limited but highly specific markers led to the separation of carcinomas, lymphoma and melanomas from other small cell tumors. Using a panel of keratin, LCA, desmin, and HMB45 is the most practical and economic approach to accurately classify these tumors $(10,31$ - 33). A second order of complimentary markers supported the initial diagnosis in most tumors and confirmed the histogenesis of few other cases. Molecular markers such as EWS-FLI1 and PAX-FKHR may be valuable for diagnostic confirma- 
tion of Ewing/PNET and rhabdomyosarcoma. Another entity named as NUT midline carcinoma should be considered if undifferentiated carcinomas with focal squamous differentiation arise in the sinonasal area $(34,35)$. This neoplasm, uniquely characterized by rearrangements of the NUT or 15q14 gene (q14;p13.1). The nuclear expression of NUT can be detected by immunohistochemistry, fluorescence in situ hybridization (FISH) or reverse transcriptase-polymerase chain reaction (RT-PCR) $(34,35)$. SRBCTs arising in the sinonasal are heterogeneous and accurate classification may be challenging due to overlapping histopathologic features. This study provides an integrative and organized approach for the application of a limited set of markers for diagnosis of these tumors.

\section{Acknowledgements}

There are no acknowledgements.

\section{Authors' Contribution}

All authors contributed equally.

\section{Financial Disclosure}

All authors declare that there is no financial disclosure.

\section{Funding Support}

All authors declare that there is no funding or support.

\section{References}

1. Bridge JA, Bowen JM, Smith RB. The small round blue cell tumors of the sinonasal area. Head Neck Pathol. 2010;4(1):84-93.

2. Kleihues P, Sobin LH. World Health Organization classification of tumors. Cancer. 2000;88(12):2887-2887.

3. Cerilli LA, Holst VA, Brandwein MS, Stoler MH, Mills SE. Sinonasal undifferentiated carcinoma: immunohistochemical profile and lack of EBV association. Am J Surg Pathol. 2001;25(2):156-63.

4. Franchi A, Moroni M, Massi D, Paglierani M, Santucci M. Sinonasal undifferentiated carcinoma, nasopharyngeal-type undifferentiated carcinoma, and keratinizing and nonkeratinizing squamous cell carcinoma express different cytokeratin patterns. Am J Surg Pathol. 2002;26(12):1597-604.

5. Perez-Ordonez B, Caruana SM, Huvos AG, Shah JP. Small cell neuroendocrine carcinoma of the nasal cavity and paranasal sinuses. Human Patholog. 1998;29(8):826-32.

6. Cohen ZR, Marmor E, Fuller GN, DeMonte F. Misdiagnosis of olfactory neuroblastoma. Neurosurg Focus. 2002;12(5).

7. Iezzoni JC, Mills SE. "Undifferentiated" small round cell tumors of the sinonasal tract: differential diagnosis update. Am J Clin Pathol. 2005;124 Suppl:S110-21.

8. Mills SE, Frierson HF, Jr. Olfactory neuroblastoma. A clinicopathologic study of 21 cases. Am J Surg Pathol.1985;9(5):317-27.

9. Khademi B, Moradi A, Hoseini S, Mohammadianpanah M. Malignant neoplasms of the sinonasal tract: report of 71 patients and literature review and analysis. Oral Maxillofac Surg. 2009;13(4):191-9.

10. Ejaz A, Wenig BM. Sinonasal undifferentiated carcinoma: clinical and pathologic features and a discussion on classification, cellular differentiation, and differential diagnosis. Adv Anat Pathol. 2005;12(3):134-43.

11. Llombart-Bosch A, Navarro S. Immunohistochemical detection of EWS and FLI-1 proteinss in Ewing sarcoma and primitive neuroectodermal tumors: comparative analysis with CD99 (MIC-2) expression. Appl Immunohistochem Mol Morphol. 2001;9(3):255-60.

12. Hicks J, Flaitz C. Rhabdomyosarcoma of the head and neck in children. Oral Oncol. 2002;38(5):450-9.

13. Morton LM, Turner JJ, Cerhan JR, Linet MS, Treseler PA, Clarke $\mathrm{CA}$, et al. Proposed classification of lymphoid neoplasms for epidemiologic research from the Pathology Working Group of the International Lymphoma Epidemiology Consortium (InterLymph). Blood. 2007;110(2):695-708.

14. Wu XC, Andrews P, Chen VW, Groves FD. Incidence of extranodal non-Hodgkin lymphomas among whites, blacks, and Asians/Pacific Islanders in the United States: anatomic site and histology differences. Cancer Epidemiol. 2009;33(5):337-46.

15. Gaal K, Sun NC, Hernandez AM, Arber DA. Sinonasal NK/T-cell lymphomas in the United States. Am J Surg Pathol. 2000;24(11):1511-7.

16. Cordes B, Williams MD, Tirado Y, Bell D, Rosenthal DI, Al-Dhahri $\mathrm{SF}$, et al. Molecular and phenotypic analysis of poorly differentiated sinonasal neoplasms: an integrated approach for early diagnosis and classification. Hum Pathol. 2009;40(3):283-92.

17. Boussen H, Khedim A, Touati S, Ben Rejeb I, Zainine R, Gritli S, et al. [Epidemiology of nasal and paranasal sinus cancer in Tunisia]. Ann Otolaryngol Chir Cervicofac. 2006;123(3):115-9.

18. Fasunla AJ, Lasisi AO. Sinonasal malignancies: a 10-year review in a tertiary health institution. J Natl Med Assoc. 2007;99(12):1407-10.

19. Gao N, Li Y, Li LJ, Wen YM. Clinical analysis of head and neck cancer cases in south-west China 1953 - 2002. I Int Med Res. 2009;37(1):189-97.

20. Gras Cabrerizo JR, Orus Dotu C, Montserrat Gili JR, Fabra Llopis JM, Leon Vintro X, De Juan Beltran J. [Epidemiologic analysis of 72 carcinomas of the nasal cavity and paranasal sinuses]. Acta Otor rinolaringol Esp. 2006;57(8):359-63.

21. Di Como CJ, Urist MJ, Babayan I, Drobnjak M, Hedvat CV, TeruyaFeldstein J, et al. p63 expression profiles in human normal and tumor tissues. Clin Cancer Res. 2002;8(2):494-501.

22. Ejaz A, Wenig BM. Sinonasal undifferentiated carcinoma: clinical and pathologic features and a discussion on classification, cellular differentiation, and differential diagnosis. Adv Anat Pathol. 2005;12(3):134-43.

23. Hedvat CV, Teruya-Feldstein J, Puig P, Capodieci P, Dudas M, Pica $\mathrm{N}$, et al. Expression of p63 in diffuse large B-cell lymphoma. Appl Immunohistochem Mol Morphol. 2005;13(3):237-42.

24. Wooff JC, Weinreb I, Perez-Ordonez B, Magee JF, Bullock MJ. Calretinin staining facilitates differentiation of olfactory neuroblastoma from other small round blue cell tumors in the sinonasal tract. Am J Surg Pathol. 2011;35(12):1786-93.

25. Bourne TD, Bellizzi AM, Stelow EB, Loy AH, Levine PA, Wick $\mathrm{MR}$, et al. p63 Expression in olfactory neuroblastoma and other small cell tumors of the sinonasal tract. Am J Clin Pathol. 2008;130(2):213-8.

26. Stevenson AJ, Chatten J, Bertoni F, Miettinen M. CD-99 (p30/p32 MIC-2) neuroectodermal/Ewing's sarcoma antigen as an immunohistochemical marker. Appl Immunohistochem Mol Morphol. 1994;2(231-4).

27. Folpe AL, Hill CE, Parham DM, O'Shea PA, Weiss SW. Immunohistochemical detection of FLI-1 protein expression: a study of 132 round cell tumors with emphasis on CD99-positive mimics of Ewing's sarcoma/primitive neuroectodermal tumor. Am J Surg Pathol.2000;24(12):1657-62.

28. Mhawech-Fauceglia P, Herrmann F, Penetrante R, Beck A, Sait S, Block AM, et al. Diagnostic utility of FLI-1 monoclonal antibody and dual-colour, break-apart probe fluorescence in situ (FISH) analysis in Ewing's sarcoma/primitive neuroectodermal tumour (EWS/PNET). A comparative study with CD99 and FLI-1 polyclonal antibodies. Histopathology. 2006;49(6):569-75.

29. Perlman EJ, Lumadue JA, Hawkins AL, Cohen K, Colombani P, Griffin CA. Primary cutaneous neuroendocrine tumors. Diagnostic use of cytogenetic and MIC2 analysis. Cancer Genet Cytogenet. 1995;82(1):30-4.

30. Thompson LD, Wieneke JA, Miettinen M. Sinonasal tract and nasopharyngeal melanomas: a clinicopathologic study of 115 cases with a proposed staging system. Am J Surg Pathol. 2003;27(5):594-611.

31. Ingeholm P, Theilgaard SA, Buchwald C, Hansen HS, Francis D. Esthesioneuroblastoma: a Danish clinicopathological study of 40 


\section{JAshraf M et al.}

consecutive cases. APMIS. 2002;110(9):639-45.

32. Chapman-Fredricks J, Jorda M, Gomez-Fernandez C. A limited immunohistochemical panel helps differentiate small cell epithelial malignancies of the sinonasal cavity and nasopharynx. Appl Immunohistochem Mol Morphol. 2009;17(3):207-10.

33. Franchi A, Miligi L, Palomba A, Giovannetti L, Santucci M. Sinonasal carcinomas: recent advances in molecular and phenotypic characterization and their clinical implications. Crit Rev Oncol Hematol. 2011;79(3):265-77.
34. Menon S, Pai P, Sengar M, Aggarwal JP, Kane SV. Sinonasal malignancies with neuroendocrine differentiation: case series and review of literature. Indian J Pathol Microbiol. 2010;53(1):28-34.

35. Davis BN, Karabakhtsian RG, Pettigrew AL, Arnold SM, French CA, Brill YM. Nuclear protein in testis midline carcinomas: a lethal and underrecognized entity. Arch Pathol Lab Med. 2011;135(11):1494-8.

36. Wenig BM.Undifferentiated malignant neoplasms of the sinonasal tract. Arch Pathol Lab Med. 2009;133(5):699-712. 